\title{
GMR
}

\section{Identification of genes related to floral organ development in pak choi by expression profiling}

\author{
H.X. Song ${ }^{1 *}$, A.M. Ping ${ }^{1 *}$, M.X. Sun ${ }^{1}$, X.H. Qi ${ }^{1}$, M.Y. Gao ${ }^{1}$, X.Y. Xu' ${ }^{1}$, \\ Z.J. Zhu' ${ }^{2}$, M.L. Li ${ }^{1}$ and L.P. Hou ${ }^{1}$ \\ ${ }^{1}$ College of Horticulture, Shanxi Agricultural University, Taigu, Shanxi, China \\ ${ }^{2}$ Key Laboratory for Quality Improvement of Agricultural Products of \\ Zhejiang Province/College of Agricultural and Food Science, \\ Zhejiang A\&F University, Hangzhou, China \\ *These authors contributed equally to this study. \\ Corresponding author: L.P. Hou \\ E-mail: sxndhlp@126.com
}

Genet. Mol. Res. 16 (1): gmr16019233

Received September 9, 2016

Accepted December 9, 2016

Published March 30, 2017

DOI http://dx.doi.org/10.4238/gmr16019233

Copyright (C) 2017 The Authors. This is an open-access article distributed under the terms of the Creative Commons Attribution ShareAlike (CC BY-SA) 4.0 License.

\begin{abstract}
Pak choi is a highly nutritious vegetable that is widely grown in China, Southeast Asia, and other parts of the world. Because it reproduces by seed, it is very important to understand the mechanism of floral organ development. Therefore, using the Chinese cabbage genome as a reference, this study analyzed the expression profiles of shoot apex genes at flower bud differentiation stages 1 and 5 , in order to identify genes related to floral organ development. The results showed that the proportion of mapped genes was high, with 84.25 and $83.80 \%$ of clean reads from the two sample saligned to the reference genome, respectively. A total of 525 differentially expressed genes (DEGs) were identified, 224 of which were upregulated and 301 were downregulated. The expression levels of genes homologous to Chinese cabbage flowering genes were also analyzed at stages 1 and 5; the expression
\end{abstract}

Genetics and Molecular Research 16 (1): gmr16019233 
levels of Bra012997 (ap1), Bra000393 (SOC1), and Bra004928 (SOC1) were significantly upregulated at stage 5 , suggesting that these three genes positively regulate floral development in pak choi. DEGs involved in floral organ development were analyzed with homologous genes from Arabidopsis thaliana; the homologous genes Bra029281 (AGL42), Bra026577 (ARPN), Bra022954 (SPL3), Bra029293 (ARF2), Bra007978 (AtRLP12), Bra033221 (SPL8), Bra008037 (LOX4), Bra001598 (IAA19), Bra003892 (PATL1), Bra038778 (AT4G21323), Bra025315 (KLCR2), and Bra013906 (DTX35) are directly related to floral organ development in Arabidopsis, suggesting that these genes have corresponding functions during flower organ development in pak choi, and could be candidates for further genetic research. These results provide a foundation for research on the molecular mechanism of flower organ development in pak choi and other Brassica rapa vegetables.

Key words: Pak choi; Floral organ; Expression profile; Gene

\section{INTRODUCTION}

Pak choi (Brassica rapa ssp chinensis 'Makino') is a crop in the botanical family Brassicaceae that originated from China. It has a wide distribution area, many varieties, and is highly nutritious. It contains glucosinolates, which have anticarcinogenic properties and are precursors of isothiocyanates. Pak choi plays an extremely important role in the production and supply of vegetable crops in China, particularly in the Yangtze River Valley and south China (Chen, 2010). Currently, the main methods used to produce F1 hybrids that take advantage of heterosis are artificial emasculation, chemical hybridization, and the use of self-incompatible and male-sterile lines (Yu, 2002). The production of hybrid seeds is difficult and expensive; therefore, it is important to understand the molecular mechanisms underlying flower organ development in pak choi.

Flowers are reproductive organs in angiosperms, and have undergone large structural changes over the course of evolution. Although flower development has been studied for over 200 years (Xu and Liu, 1998), research on floral organs in traditional biology has mainly focused on morphological descriptions and the physiological mechanism of flowering. Beginning in the 1980s through research on floral mutants of Arabidopsis and Antirrhinum, our understanding of the molecular mechanisms of floral organ development has advanced significantly with the development of molecular genetics, and has become an attractive "hotspot" in the field of plant developmental biology (Xu, 1999). Since the ABC model of flower development was first presented by Coen and Meyerowitz (1991), the key genes related to floral organ development have been successfully cloned from a variety of plants, and the regulation of flower development is now well understood, which has taken research on flower development from the ecological, physiological, and biochemical levels to the molecular genetic level. With the discovery of new mutants, the genetic model of flower organ development has been continuously updated and improved, progressing from the $\mathrm{ABC}$ model to the $\mathrm{ABCDE}$ and four factors models (Tang et al., 2013).

Recently, with the application of molecular biology in many areas of reproductive biology, the molecular mechanism of flower development in Brassica rapa has attracted more

Genetics and Molecular Research 16 (1): gmr16019233 
and more attention. It has been recognized that flower development in B. rapa is a complex process that is regulated by multiple genes. Taking male sterility as a starting point, several studies of pollen development genes in pak choi have been conducted since 2001 (Han, 2011). A series of genes related to pollen development have been isolated, including CYP86MF (Yu et al., 2004), BcMF1 (Wang, 2003), BcMF2 (Wang et al., 2005), BcMF3 and BcMF4 (Liu et al., 2007), BcMF5 and BcMF6 (Zhang et al., 2008), BcMF7 (Huang et al., 2007), BcMF8 (Huang et al., 2008a), BcMF9 (Huang et al., 2009), BcMF10 (Huang et al., 2008b), BcMF11 (Song et al., 2007), BcMF12, BcMF13 (Li et al., 2008), BcMF19 (Huang et al., 2011), BcMF22 (Li, 2011), BcMFJMJ30, BcBGAL11, and MIR158 (Jiang, 2014), BcMF20 (Han, 2011), $B c A H A 8$ (Qiu, 2012) and BcSKS11 (Zhang, 2011), BcMYB, and BjMF6. The functions of some of these genes have been described, including the cytochrome P450 gene CYP86MF, the pectinesterase gene $B c M F 3$, the polygalacturonase genes $B c M F 2, B c M F 6$, and $B c M F 9$, the arabinogalactan protein genes $B c M F 8$ and $B c M F 18$, the beta-galactosidase gene $B c B G A L 11$, and the pollen wall protein gene $B c M F 5$. However, no research has been conducted on floral organ genes in pak choi. With the rapid development of bioinformatics, the genomes of the model plants Arabidopsis and Chinese cabbage have been sequenced, and genomic data are continuously accruing, which enable studies on gene expression and the regulation of flower organ development in pak choi (Qiu, 2012). Therefore, using the Chinese cabbage genome as a reference, this study compared gene expression profiles at flower bud differentiation stages 1 and 5 to identify genes associated with floral organ development, which will establish a foundation for further research into reproductive development in B. rapa ssp chinensis.

\section{MATERIAL AND METHODS}

\section{Plant materials and RNA extraction}

The pak choi inbred line '75\#' that bolts easily was used in our experiments. This line was obtained from the Institute of Vegetable Research, Shanxi Academy of Agricultural Sciences. Germinating seeds were maintained at $4^{\circ} \mathrm{C}$ for 20 days for the low temperature treatment, after which, seedlings with cotyledons were transplanted to a tray containing soil. Traditional field management was practiced, and flower bud differentiation was observed. The shoot apices of flower buds at differentiation stages 1 and 5 were sampled on days 16 and 17 after transplanting (Song et al., 2015); these samples, each weighed $0.15 \mathrm{~g}$, were named V16 and V17, respectively, and were used for the extraction of total RNA. Total RNA was extracted from each sample using an RNeasy Plant Mini Kit (Qiagen, Cat. No. 74903) following the manufacturer's instructions. The extracted RNA was stored at $-80^{\circ} \mathrm{C}$ for subsequent analysis. cDNA library construction, sequencing, gene expression analysis, and functional annotation was conducted by Biomarker Technologies Co. Ltd. The methods of DNA sequencing, gene expression analysis, and functional annotation were previously described by Sun et al. (2015).

\section{Screening of differentially expressed genes (DEGs) related to floral organ development}

DEGs that were potentially related to floral organ development were screened by Gene Ontology (GO) annotation and Kyoto Encyclopedia of Genes and Genomes (KEGG) pathway analysis. In addition, the flowering genes from Chinese cabbage published in BRAD (http://brassicadb.org) were compared with the profiles to identify genes involved in flower

Genetics and Molecular Research 16 (1): gmr16019233 
organ development in pak choi. These screened genes were compared to homologous genes from Arabidopsis to predict their function in pak choi flowering.

\section{Quantitative real-time polymerase chain reaction (RT-qPCR)}

To validate the RNA-Seq results, a RT-qPCR was performed using gene-specific primers for four randomly selected genes. For reverse transcription, the concentration of each RNA sample was adjusted to $500 \mathrm{ng} / \mu \mathrm{L}$. Single-stranded cDNA was synthesized using a PrimeScript ${ }^{\mathbb{}}$ RT reagent kit (Perfect Real Time; TaKaRa, Cat. No. DRR037A) following the manufacturer's instructions. Specific primers used for RT-qPCR were designed using Primer 3 software (http://primer3.ut.ee/) based on the gene sequence, and the pak choi ACTIN gene was chosen as an internal reference gene. These four primers were all adjusted to $10 \mu \mathrm{M}$. RTqPCR was conducted using SYBR ${ }^{\circledR}$ Premix Ex Taq ${ }^{\mathrm{TM}}$ II (Tli RNaseH Plus; TaKaRa, Cat. No. $\mathrm{RR} 820 \mathrm{~A})$ in a $25-\mu \mathrm{L}$ reaction volume. The reaction mixture included $20 \mathrm{ng}$ cDNA $(2 \mu \mathrm{L}), 8.5$ $\mu \mathrm{L} \mathrm{ddH_{2 }} \mathrm{O}, 12.5 \mu \mathrm{L} \mathrm{SYBR}^{\circledR}$ mix, and $2 \mu \mathrm{L}$ primers. The thermocycler program was as follows: $95^{\circ} \mathrm{C}$ for $1 \mathrm{~min}$, followed by 40 cycles at $95^{\circ} \mathrm{C}$ for $15 \mathrm{~s}, 55^{\circ} \mathrm{C}$ for $30 \mathrm{~s}$, and $72^{\circ} \mathrm{C}$ for $30 \mathrm{~s}$. Realtime PCR analysis was performed in an ABI 7500 apparatus and the relative expression level was calculated using the $\Delta \Delta \mathrm{Ct}$ method (Stanko et al., 2014).

\section{Data access}

The transcriptome sequencing data from this study have been deposited in the National Center for Biotechnology Information Sequence Read Archive database, and are accessible through accession number SRP075755 (http://www.ncbi.nlm.nih.gov/sra).

\section{RESULTS}

\section{Quality assessment of the sequencing results}

Gene expression profiles were analyzed using RNA-Seq technology. As shown in Table 1, 10,709,754 and 10,333,666 reads were obtained from the V16 and V17 libraries, respectively. Clean reads obtained by filtering out adaptor sequences, contaminating sequences, and low-quality reads accounted for $99.86 \%$ (V16) and $99.84 \%$ (V17) of the total reads, meaning that the quality of the sequencing was excellent.

\section{Table 1. Illumina DNA sequencing reads from the two datasets and mapping results.}

\begin{tabular}{l|c|c}
\hline Read type & Stage 1 & Stage 5 \\
\hline Total read & $10,709,754$ & $10,333,666$ \\
\hline Clean read & $10,695,085$ & $10,317,519$ \\
\hline Mapped reads & $9,010,384$ & $8,645,982$ \\
\hline Unique-mapped reads & $8,454,786$ & $8,113,061$ \\
\hline Multiple-mapped reads & 555,598 & 532,921 \\
\hline
\end{tabular}

In order to obtain gene expression information, high-quality reads from the two libraries were aligned to the Chinese cabbage genome sequence. A total of 84.25 and $83.80 \%$ of the clean reads from the V16 and V17 libraries, respectively, were mapped to the reference genome, including unique-mapped reads and multiple-mapped reads. The proportion of

Genetics and Molecular Research 16 (1): gmr16019233 
mapped genes was high, indicating that the sequences and reference genome were suitable for further analysis. The unique-mapped reads accounted for 93.83 and $93.84 \%$ of the total mapped reads in the V16 and V17 libraries, respectively, so could be used for further analysis.

\section{Screening of DEGs}

The unique reads that aligned perfectly to Chinese cabbage genes from the two shoot apex libraries at flower bud differentiation stages 1 (V16) and 5 (V17) were used for DEG analysis (Table 2). A total of 525 DEGs were identified, 224 and 301 of which were upregulated and downregulated, respectively. DEGs in which the fold change was between 2 and 10 comprised the majority (474), and included 194 genes that were upregulated and 280 that were downregulated. The expression fold changes for 47 genes were between 10 and 50, with 26 upregulated and 21 downregulated. Only four genes exhibited a $>50$-fold change, and all of them were upregulated.

Table 2. Characteristics of the differentially expressed genes (DEGs).

\begin{tabular}{l|l|c}
\hline DEG fold change & \multicolumn{2}{|c}{} \\
\hline \multirow{3}{*}{$>$} & Total & Number of DEGs between stages 1 and 5 \\
\cline { 2 - 3 } & Up & 525 \\
\cline { 2 - 3 } & Down & 224 \\
\hline \multirow{3}{*}{-10} & Total & 401 \\
\cline { 2 - 3 } & Up & 194 \\
\cline { 2 - 3 } & Down & 280 \\
\hline \multirow{2}{*}{$10-50$} & Total & 47 \\
\cline { 2 - 3 } & Up & 26 \\
\cline { 2 - 3 } & Down & 21 \\
\cline { 2 - 3 } & Total & 4 \\
\cline { 2 - 3 } & Up & 4 \\
\cline { 2 - 3 } & Down & 0 \\
\cline { 2 - 3 } & &
\end{tabular}

\section{GO functional analysis of DEGs}

For the GO enrichment analysis, a GO term with a corrected $\mathrm{P}$ value of $\leq 0.05$ was considered significantly enriched. Figure 1 shows that the numbers of significantly enriched terms in cell composition, molecular function, and biological process were 4, 9, and 24, respectively. The GO enrichment analysis revealed that the proteins encoded by these DEGs were mainly located in the apoplast, extracellular regions, and cell wall, and had the molecular functions of hydrolase activity, xyloglucosyl transferase activity, and acid phosphatase activity, which are involved in the biological processes of glucose metabolism, phenylalanine metabolism, the low temperature response, and the gibberellic acid (GA) response.

\section{KEGG pathway analysis of DEGs}

The DEGs were annotated and classified in the KEGG database. The main biochemical metabolism and signal transduction pathways in which the DEGs were involved were identified by enrichment analysis. The 525 DEGs were mapped to referable and canonical KEGG pathways. Overall, these genes were distributed in 64 metabolic pathways, but only the following five were significant: flavonoid biosynthesis, phenylpropanoid biosynthesis, linoleic acid metabolism, arginine and proline metabolism, and phenylalanine metabolism

Genetics and Molecular Research 16 (1): gmr16019233 
(Table 3), suggesting that these pathways play an important role in the process of flower bud differentiation from stage 1 to stage 5 in pak choi.

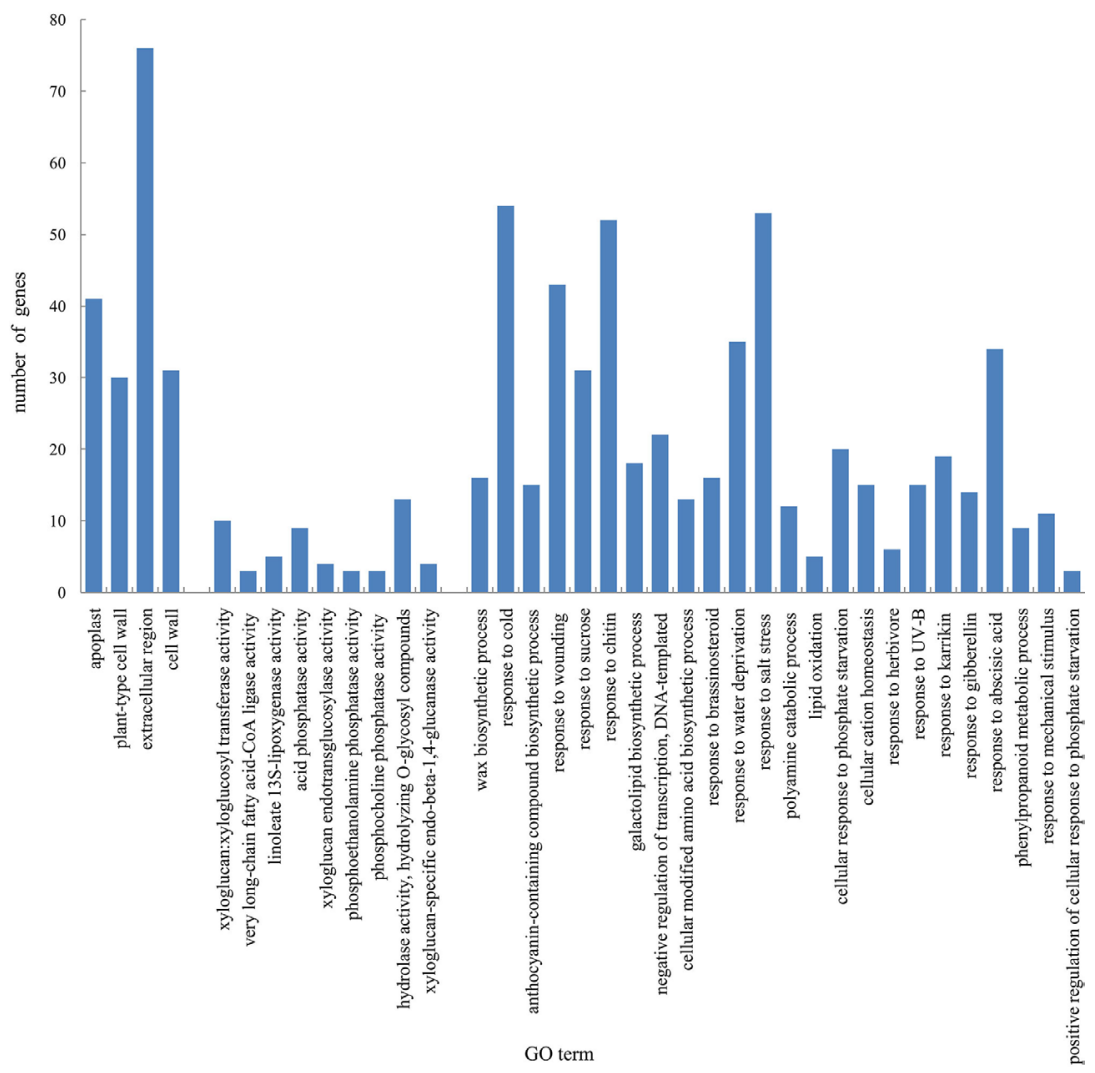

Figure 1. Gene Ontology (GO) enrichment analysis of differentially expressed genes (DEGs). A corrected P value threshold of $\leq 0.05$ was used to identify significantly enriched GO terms in the DEGs.

Table 3. Kyoto Encyclopedia of Genes and Genomes enrichment analysis of differentially expressed genes.

\begin{tabular}{l|c|c|c}
\hline Pathway & ID & Frequency & Corrected P value \\
\hline Flavonoid biosynthesis & ko00941 & 8 & $2.13 \mathrm{E}-05$ \\
\hline Phenylpropanoid biosynthesis & ko00940 & 13 & 0.000228 \\
\hline Linoleic acid metabolism & ko00591 & 5 & 0.000505 \\
\hline Arginine and proline metabolism & ko00330 & 9 & 0.014962 \\
\hline Phenylalanine metabolism & ko00360 & 9 & 0.041315 \\
\hline
\end{tabular}

\section{Correspondence analysis of Chinese cabbage flower genes with the expression profiles of pak choi}

The expression of Chinese cabbage flowering genes in the BRAD database was analyzed at flower bud differentiation stages 1 and 5 in pak choi (Table 4). We found that the homologous gene expression of Bra012997 (ap1), Bra000393 (SOC1), and Bra004928

Genetics and Molecular Research 16 (1): gmr16019233 
Table 4. Correspondence analysis of Chinese cabbage flower genes and pak choi expression profiles.

\begin{tabular}{|c|c|c|c|c|c|}
\hline \multirow[t]{2}{*}{ \# } & \multirow[t]{2}{*}{ Gene name } & \multirow[t]{2}{*}{ Gene ID } & \multicolumn{2}{|c|}{ RPKM } & \multirow[t]{2}{*}{ Up or Down } \\
\hline & & & Stage 1 & Stage 5 & \\
\hline 1 & $A G L 24$ & Bra019221 & 122.9 & 115.7 & $\downarrow$ \\
\hline 2 & $A G L 24$ & Bra013891 & 0 & 0 & $\rightarrow$ \\
\hline 3 & apl & Bra038326 & 1.7 & 2.3 & $\uparrow$ \\
\hline 4 & apl & Bra004007 & 0.6 & 3.3 & $\uparrow$ \\
\hline 5 & apl & Bra011021 & 8.1 & 4.0 & $\downarrow$ \\
\hline 6 & apl & Bra029347 & 1.6 & 2.5 & $\uparrow$ \\
\hline 7 & apl & Bra012997 & 3.7 & 14.4 & $\uparrow$ \\
\hline 8 & apl & Bra035952 & 1.1 & 4.8 & $\uparrow$ \\
\hline 9 & apl & Bra004361 & 4.2 & 12.0 & $\uparrow$ \\
\hline 10 & $A P 2$ & Bra011741 & 24.3 & 36.4 & $\uparrow$ \\
\hline 11 & $A P 2$ & Bra017809 & 24.4 & 32.7 & $\uparrow$ \\
\hline 12 & ARP6 & - & - & - & 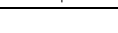 \\
\hline 13 & $C D F 1$ & Bra029261 & 6.9 & 5.5 & $\downarrow$ \\
\hline 14 & $C D F 1$ & Bra010082 & 3.2 & 2.4 & $\downarrow$ \\
\hline 15 & $\mathrm{CO}$ & Bra008669 & 0.3 & 0 & $\downarrow$ \\
\hline 16 & $\mathrm{CO}$ & Bra023541 & 0.5 & 1.3 & $\uparrow$ \\
\hline 17 & COPI & Bra005541 & 12.1 & 13.2 & $\uparrow$ \\
\hline 18 & COP1 & Bra021818 & 0 & 0 & $\rightarrow$ \\
\hline 19 & CRY1 & $\mathrm{Bra} 030568$ & 39.7 & 43.6 & $\uparrow$ \\
\hline 20 & $C R Y I$ & Bra015313 & 6.8 & 5.2 & $\downarrow$ \\
\hline 21 & CRY2 & Bra037880 & 67.9 & 53.7 & $\downarrow$ \\
\hline 22 & CSTF64 & Bra003913 & 8.3 & 10.4 & $\uparrow$ \\
\hline 23 & CSTF64 & Bra007985 & 4.4 & 6.7 & $\uparrow$ \\
\hline 24 & CSTF77 & Bra016588 & 6.8 & 10.4 & $\uparrow$ \\
\hline 25 & DHF & - & - & - & - \\
\hline 26 & $F C A$ & Bra038446 & 7.8 & 10.2 & $\uparrow$ \\
\hline 27 & $F D$ & Bra010504 & 5.7 & 7.7 & $\uparrow$ \\
\hline 28 & $F D$ & Bra011648 & 13.7 & 24.2 & $\uparrow$ \\
\hline 29 & $F D$ & Bra017735 & 7.1 & 4.5 & $\downarrow$ \\
\hline 30 & $F K F 1$ & Bra038831 & 7.1 & 9.9 & $\uparrow$ \\
\hline 31 & $F K F 1$ & Bra038830 & 9.9 & 10.1 & $\uparrow$ \\
\hline 32 & $F K F 1$ & Bra038832 & 0.1 & 0.1 & $\rightarrow$ \\
\hline 33 & $F L C$ & Bra009055 & 31.6 & 23.9 & $\downarrow$ \\
\hline 34 & $F L C$ & Bra028599 & 17.3 & 12.3 & $\downarrow$ \\
\hline 35 & $F L C$ & Bra006051 & 14.1 & 16.2 & $\uparrow$ \\
\hline 36 & $F L C$ & Bra022771 & 2.6 & 2.6 & $\rightarrow$ \\
\hline 37 & $F L D$ & Bra001357 & 10.2 & 9.6 & $\vec{\downarrow}$ \\
\hline 38 & $F L K$ & Bra001111 & 52.7 & 44.8 & $\downarrow$ \\
\hline 39 & FLM & - & - & - & \\
\hline 40 & $F P A$ & Bra004761 & 9.9 & 10.7 & $\uparrow$ \\
\hline 41 & FRI & Bra035723 & 3.3 & 2.7 & $\downarrow$ \\
\hline 42 & $F T$ & Bra022475 & 0 & 0 & $\rightarrow$ \\
\hline 43 & $F T$ & Bra004117 & 0.4 & 0 & $\downarrow$ \\
\hline 44 & $F T$ & Bra015710 & 0 & 0 & $\rightarrow$ \\
\hline 45 & $F T$ & Bra010052 & 0 & 0 & $\rightarrow$ \\
\hline 46 & $F V E$ & Bra031085 & 53.9 & 50.2 & $\downarrow$ \\
\hline 47 & $F V E$ & Bra040678 & 43.7 & 42.3 & $\downarrow$ \\
\hline 48 & $F V E$ & Bra036717 & 54.6 & 49.9 & $\downarrow$ \\
\hline 49 & $F V E$ & Bra011133 & 4.1 & 4.1 & $\rightarrow$ \\
\hline 50 & $F V E$ & Bra040681 & 1.2 & 0.8 & $\downarrow$ \\
\hline 51 & $F Y$ & Bra006202 & 13.2 & 16.1 & $\uparrow$ \\
\hline 52 & $F Y$ & $\mathrm{Bra} 023416$ & 10.5 & 11.5 & $\uparrow$ \\
\hline 53 & GAI & Bra036239 & 0.9 & 0.6 & $\downarrow$ \\
\hline 54 & GAI & Bra000864 & 0.3 & 0 & $\downarrow$ \\
\hline 55 & GAI & Bra024875 & 153.9 & 146.8 & $\downarrow$ \\
\hline 56 & GAI & Bra017443 & 173.6 & 136.7 & $\downarrow$ \\
\hline 57 & $G I$ & Bra024536 & 11.2 & 13.0 & $\uparrow$ \\
\hline 58 & GIDIA & Bra039460 & 27.3 & 26.9 & $\downarrow$ \\
\hline 59 & GIDIA & Bra009970 & 17.0 & 19.4 & $\uparrow$ \\
\hline 60 & GIDIA & Bra003520 & 0.5 & 1.1 & $\uparrow$ \\
\hline
\end{tabular}

RPKM, reads per kb per million reads; $\uparrow$, upregulated; $\downarrow$, downregulated; $\rightarrow$, unchanged.

Genetics and Molecular Research 16 (1): gmr16019233 
(SOC1) was significantly upregulated at stage 5. In A. thaliana, ap1 encodes a MADS-box protein involved in flowering that regulates the expression of $S O C 1$, and is also upregulated by SOC1. In addition to Bra011021, the expression levels of four other ap1 genes (Bra038326, Bra004007, Bra029347, and Bra004361) all increased, although they were not as high as that of Bra012997. Therefore, apl genes may also be involved in the regulation of flowering in pak choi. SOCl controls flowering, and is required for $C O$ to promote flowering. SOCl acts downstream of FT. The overexpression of SOC1 suppresses not only late flowering in plants that have functional $F R I$ and $F L C$ alleles, but also delays phase transitions during the vegetative stages of development in A. thaliana. In addition to the expression of Bra004928 and Bra000393 being significantly upregulated, the other SOCl genes (Bra004927 and Bra000392) were also upregulated, whereas Bra039324 was slightly downregulated. Therefore, SOC1 is also predicted to promote flowering in pak choi.

When other Chinese cabbage flowering genes were analyzed, five genes related to floral organ development were found; these included $A P 2, G I D 1 A, G I D 1 B, G I D 1 C$, and $P R C 2 . A P 2$ is involved in the specification of floral organ identity in $A$. thaliana. The expression of two $A P 2$ genes, Bra011741 and Bra017809, increased at stage 5 . GIDIA, GID1B, and GIDIC encode a GA receptor ortholog of the rice GA receptor gene, which is involved in floral organ morphogenesis. In addition to the slightly decreased expression of Bra039460 and unchanged expression of Bra040420, the expression levels of Bra009970, Bra003520, and Bra007722 were upregulated (Table 4). PRC2 encodes a polycomb group protein with a zinc-finger domain that is involved in the regulation of reproductive development. PRC2 forms a complex with FIE, CLF, and MSI1 that modulates the expression of target genes including $A G, P I$, and $A P 3$. The expression of the homologous genes Bra032169, Bra015200, and Bra022541 all increased during flower bud differentiation in pak choi. Therefore, the pak choi homologs of Bra011741, Bra017809, Bra009970, Bra003520, Bra007722, Bra032169, Bra015200, and Bra022541 may also be involved in floral organ development.

In addition to the above Chinese cabbage flowering genes, there were genes related to light (light cycle, quality, signal transduction, and photomorphogenesis), such as $C D F 1$, CO, COP1, TEM1, CRY1, CRY2, LHP1, PHYA, PHYB, and SPA, the floral transition-related genes $F K F 1, F C A, F L C, F L D, C S T F 64$, and CSTF77, the flowering-related genes FD, FLK, $F P A, F r i, F T, F V E$, and $F Y$, the GA-related genes GAI, GAI and GI, RGA, SPY, and the vernalization-related genes VIN3 and VRN2. The expression patterns of these genes were mostly consistent with those seen in Chinese cabbage during flower bud differentiation, indicating that the genetic mechanisms underlying floral development are similar in Chinese cabbage and pak choi.

\section{Comparison of DEGs related to floral organ development with their homologs in A. thaliana}

By analyzing the gene expression profiles, it was found that there were 35 DEGs related to floral organ development between flower bud differentiation stages 1 and 5 (Table 5). Of these, 22 were upregulated and 13 were downregulated, and three genes coincided with flowering genes in Chinese cabbage: Bra000393 (SOC1), Bra004928 (SOC1), and Bra012997 (ap1). The homologs of these genes from Chinese cabbage have been published, and are described in the previous section.

Genetics and Molecular Research 16 (1): gmr16019233 
Table 5. Correspondence analysis of differentially expressed genes related to floral organ development in pak choi and their homologs in Arabidopsis thaliana.

\begin{tabular}{|c|c|c|c|c|}
\hline Gene ID & $\log _{2} \mathrm{FC}$ & nr_annotation & $\begin{array}{l}\text { Chinese cabbage } \\
\text { floral gene }\end{array}$ & $\begin{array}{c}\text { Arabidopsis gene or } \\
\text { annotation }\end{array}$ \\
\hline Bra000393 & 1.764137 & MADS-box protein $A G L 20$ & SOC1 & SOCl \\
\hline Bra000568 & -1.43187 & Predicted protein & - & TPS10 \\
\hline Bra001598 & -2.07617 & Auxin-responsive protein $I A A I 9$ & - & IAA19 \\
\hline Bra003892 & -1.37326 & Hypothetical protein & - & PATL1 \\
\hline Bra004836 & 1.62323 & Beta glucosidase 15 & - & $B G L U 13$ \\
\hline Bra004928 & 1.381429 & MADS-box protein & SOC1 & SOC1 \\
\hline Bra007978 & 1.289333 & Hypothetical protein & - & $R L P 12$ \\
\hline Bra008037 & -1.27487 & Lipoxygenase 4 & - & $L O X 4$ \\
\hline Bra011184 & 1.38791 & Diacylglycerol kinase 7 & - & $D G K 7$ \\
\hline Bra012997 & 1.917994 & Agamous-like MADS-box protein $A G L 8$ homolog & apl & $A G L 8$ \\
\hline Bra013906 & 1.57119 & Hypothetical protein & - & DTX35 \\
\hline Bra017839 & -1.16143 & BTB and TAZ domain protein 5 & - & BT5 \\
\hline Bra018412 & 5.713405 & Hypothetical protein & - & WSD1 \\
\hline Bra020349 & -4.69827 & Subtilase family protein & - & AT5G58830 \\
\hline Bra020816 & 1.63828 & Uncharacterized protein & - & AT4G23530 \\
\hline Bra022161 & 1.165676 & Hypothetical protein & - & $J R 1$ \\
\hline Bra022954 & 2.29602 & Hypothetical protein & - & SPL3 \\
\hline Bra024749 & 1.373295 & Hypothetical protein & - & CUT1 \\
\hline Bra024848 & -1.58058 & Endoxyloglucan transferase & - & ATXTH27 \\
\hline Bra025315 & -1.14993 & Hypothetical protein & - & $K L C R 2$ \\
\hline Bra025683 & -1.29451 & Uncharacterized protein & - & ATIG18740 \\
\hline Bra025914 & -1.87012 & Eprl & - & EPRI \\
\hline Bra026577 & 1.401154 & Predicted protein & - & $A R P N$ \\
\hline Bra028286 & 2.932274 & Hypothetical protein & - & AT5G51950 \\
\hline Bra029224 & -2.94055 & Cytochrome P450 & - & CYP94BI \\
\hline Bra029281 & 1.491943 & Protein agamous-like 42 & - & $A G L 42$ \\
\hline Bra029293 & -1.22053 & Auxin response factor $2-1$ & - & $A G E 1$ \\
\hline Bra030284 & 1.835634 & Glycine-rich RNA-binding protein GRP2A & - & GRP7 \\
\hline Bra031210 & 1.183685 & Glycine-rich RNA-binding protein $G R P 1 A$ & - & GRP7 \\
\hline Bra031249 & 1.437032 & Hypothetical protein & - & $A B C G 15$ \\
\hline Bra032670 & 2.095754 & Protein ECERIFERUM 1 & - & CERI \\
\hline Bra033221 & 1.857669 & SQUAMOSA-promoter binding protein-like 8 & - & SPL8 \\
\hline Bra038778 & 1.043807 & Hypothetical protein & - & AT4G21323 \\
\hline Bra039702 & 1.676074 & Epithiospecifier protein & - & TASTY \\
\hline Bra040837 & -2.07617 & Hypothetical protein & - & AT4G27890 \\
\hline
\end{tabular}

FC, fold change.

Another 32 genes were analyzed by comparing them with their homologs in $A$. thaliana. The Bra001598 homolog is a primary auxin-responsive gene that is involved in the regulation of stamen filament development in Arabidopsis, which is similar to its function in pak choi. Bra003892 is probably involved in pollen tube growth and glycolysis in pak choi; the homologous gene PATL1 encodes a novel cell-plate-associated protein that is involved in pollen tube growth in Arabidopsis. Bra007978 is presumed to play a role in stamen development and the jasmonic acid-mediated signaling pathway in pak choi, while the homologous gene RLP12 in Arabidopsis encodes a CLAVATA2 (CLV2)-related protein that regulates both meristem and organ development. It is predicted that Bra008037 is involved in stamen filament development, pollen development, and anther dehiscence in pak choi, because its homolog LOX4 encodes a PLAT/LH2 domain-containing lipoxygenase family protein in Arabidopsis that has a similar function in pak choi. It is predicted that Bra011184 is involved in pollen tube growth in pak choi; the homologous gene in Arabidopsis encodes a diacylglycerol kinase, and applying a specific diacylglycerol kinase inhibitor to growth media results in reduced root elongation and plant growth. It is expressed throughout the plant, but 
expression is strongest in flowers and young seedlings; although it does not have a function that is directly related to floral organ development, decreases in root and plant growth would probably impair the development of floral organs. Bra013906 is predicted to be involved in pollen development, anther dehiscence, pollen tube growth, and flavonoid metabolism in pak choi. Its homolog DTX35 has a similar function in flavonoid metabolism, pollen development, and pollen release and viability in Arabidopsis. Bra020349 is predicted to play a role in the development of stamens and petals, because the homologous gene in Arabidopsis is expressed in the embryo sac central cell, the plant embryo, and plant sperm cells. SPL3, the homolog of Bra022954, encodes a member of the SPL (SQUAMOSA-promoter binding protein-like) family, may directly regulate $A P 1$, and is involved in the regulation of flowering and the vegetative phase change. Bra025315 and the homologous gene in Arabidopsis have similar functions in pollen tube growth. Bra026577 is predicted to regulate flower development, and is involved in anther development and pollination. Its homolog in A. thaliana encodes plastacyanin, a blue copper protein, which is involved in anther development and pollination, and is expressed in the transmitting tract of the pistil. Bra029281 is predicted to regulate flower development, because its Arabidopsis homolog, AGL24, also regulates floral development, and is expressed during the floral transition phase. The Bra029293 homolog encodes an auxin response factor and positively regulates flower development, which is consistent with its function in pak choi. Bra033221 is predicted to be involved in micro- and megasporogenesis and anther development in pak choi; micro-and megasporogenesis, trichome formation on sepals, and stamen filament elongation are affected in mutants of its homolog in Arabidopsis, SPL8. SPL8 is a member of the SPL gene family in Arabidopsis, and encodes an SBP-box protein. Bra038778 is thought to participate in cell wall composition and pollen tube growth in pak choi. Its homolog in Arabidopsis is expressed in pollen tube cells and is involved in pollen tube growth, which is consistent with its function in pak choi.

In addition, our data demonstrate that Bra000568, Bra017839, and Bra039702 probably participate in the specification of floral organ identity; Bra004836, Bra020816, Bra024749, and Bra025683 probably participate in pollen tube development; and Bra018412 and Bra031249 may participate in petal morphogenesis in pak choi. Bra022161, Bra028286, and Bra040837 are probably involved in stamen development; Bra024848 may regulate stamen filament development; Bra025914 may play a role in the regulation of flower development; and Bra029224, Bra030284, Bra031210, and Bra032670 are probably involved in anther development, although their homologous genes in Arabidopsis have no similar functions with respect to floral organ development.

Based on the results of the above analysis, most of the DEGs had functions related to floral development. Some genes act in stamen filament and pollen development, some in flower transition, and some in gamete formation. The Arabidopsis homologs of Bra001598, Bra003892, Bra007978, Bra008037, Bra011184, Bra013906, Bra020349, Bra022954, Bra025315, Bra026577, Bra029281, Bra029293, Bra033221, and Bra038778 have functions directly related to floral organ development; therefore, we predict that these genes have corresponding functions during the process of flower organ development in pak choi, and need to be studied further.

\section{Validation of DEGs by RT-qPCR}

To validate the RNA-Seq data, Bra022954, Bra000393, Bra004928, and Bra033221

Genetics and Molecular Research 16 (1): gmr16019233 
were selected for RT-qPCR analysis at flower bud differentiation stages 1 and 5 (Figure 2). The expression patterns of these genes obtained by RT-qPCR and RNA-Seq were similar, indicating that the results obtained from the RNA-Seq were reliable.
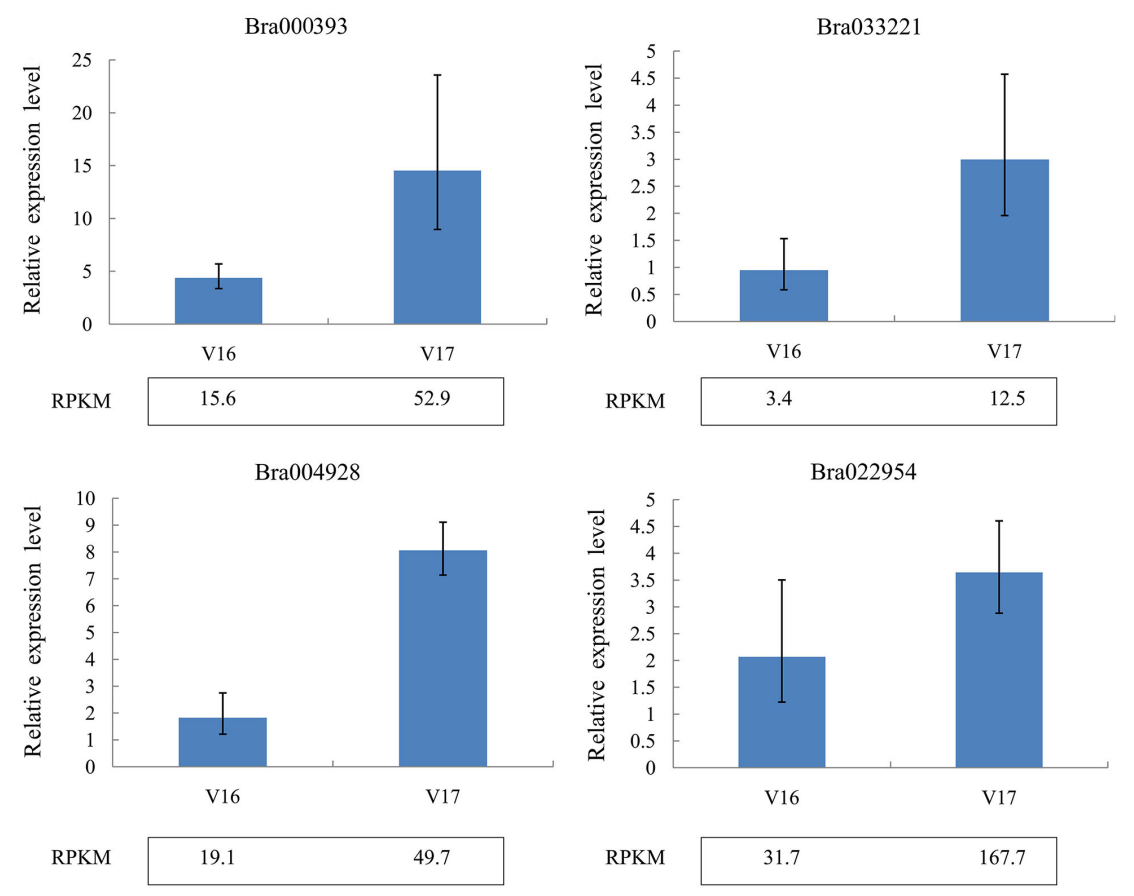

Figure 2. Quantitative real-time polymerase chain reaction validation of RNA-Seq results based on gene expression level. RPKM, reads per kb per million reads. Columns with error bars indicate relative quantification (RQ); RQmax and RQmin were calculated using an ABI 7500.

\section{DISCUSSION}

Previous research has shown that cells divide and differentiate into a layer of four sporophytic cells in the early stage of anther development, and that the mutation of early genes will cause a male-sterile phenotype (Scott et al., 2004). SPL/NZZ is a MADS-box transcription factor that is involved in the isolation and differentiation control of early cell differentiation in Arabidopsis. In SPL/NZZ mutant anthers, the formation of the archesporial cells is normal, but the primary sporogenous cells fail to differentiate into microsporocytes and the anther wall. The same problem also appears in the pistil mutant; therefore, either male or female SPL mutants are sterile (Schiefthaler et al., 1999; Yang et al., 1999). Therefore, SPL/NZZ plays an important role in the process of flower organ development. SPL8 is a member of the SBP-box protein family. Studies in Arabidopsis have shown that SPL8 mutants exhibit a strong reduction in fertility. This reduced fertility is primarily attributable to abnormally developed microsporangia, which exhibit premeiotic abortion of the sporocytes (Unte et al., 2003). Another study showed that the constitutive overexpression of SPL 8 affects fertility due to non-dehiscent anthers, which probably result from a constitutive GA response, suggesting a positive role for SPL8 in GA-mediated anther development (Zhang et al., 2007b). The results

Genetics and Molecular Research 16 (1): gmr16019233 
of the present study suggest that the SPL8 homolog Bra033221 is involved in micro- and megasporogenesis and anther development in pak choi; its expression was more upregulated in flower bud differentiation stage 5 than in stage 1 , which is consistent with previous results in A. thaliana. This suggests that Bra033221 has similar functions as the $A$. thaliana SPL8 gene in pak choi. However, the ways in which Bra033221 affects microspore development need further clarification. SPL3 is also a member of the SBP-box protein family. Yamaguchi et al. (2009) suggested that $S P L 3$ acts together with other microRNA-regulated $S P L$ transcription factors to control the timing of flower formation. Cardon et al. (1997) suggested that SPL3 recognizes a conserved sequence motif in the promoter region of the $A$. thaliana floral meristem identity gene $A P 1$. Similar to $A P 1$, the constitutive expression of $S P L 3$ results in early flowering. In our study, the expression of the SPL3 homolog Bra022954 was more upregulated in flower bud differentiation stage 5 than in stage 1 , which is consistent with previous results in $A$. thaliana.

Flavonoids are a large class of important secondary metabolites in plants. Besides providing pigmentation to flowers, fruits, seeds, and leaves, flavonoids also play key roles in signaling between plants and microbes, in male fertility in some species, in defense as antimicrobial agents and feeding deterrents, and in ultraviolet protection (Winkel-Shirley, 2001). DTX35 is a gene related to flavonoid metabolism in A. thaliana. Another study showed that FFT (AtDTX35) is highly transcribed in floral tissues. Mutant analysis has demonstrated that the absence of $F F T$ transcripts affects flavonoid levels in the plant, and that altered flavonoid metabolism has wide-ranging consequences; root growth, seed development and germination, and pollen development, release, and viability are all affected (Thompson et al., 2010). In our experiment, flavonoid metabolism was significantly enriched in flower bud differentiation stages 1 to 5, indicating that flavonoid metabolism is closely related to the development of floral organs in pak choi. Bra013906, which is homologous to AtDTX35, is predicted to function in pollen development, anther dehiscence, pollen tube growth, and flavonoid metabolic processes in pak choi, which is consistent with previous results in A. thaliana.

Phenylpropanoids are a diverse class of plant natural compounds that are composed of a benzene ring connected to a three-carbon propene tail, and are members of a larger class of phenolic compounds. In this study, phenylpropanoid biosynthesis was significantly enriched in the process of floral organ development. Although no studies have found a direct relationship between phenylpropanoids and floral organ development, some have shown that the biosynthesis of flavonoid pigments in flowers requires the coordinated expression of genes that encode enzymes in the phenylpropanoid biosynthetic pathway (Sablowski et al., 1994). Moreover, flavonoid pigments have a direct relationship with floral organ development. Therefore, it is reasonable to assume that phenylpropanoid biosynthesis is significantly enriched during the development of floral organs in pak choi.

Linoleic acid is a natural antioxidant (Zhang et al., 2007a). The oleic acid dehydrogenase gene is an important functional gene that catalyzes the conversion of oleic acid to linoleic acid in peanuts. Research has shown that the relative expression level of the oleic acid dehydrogenase gene is low in roots, stems, and leaves of the peanut, while its expression is significantly higher in flowers than in other organs, which indirectly indicates that the linoleic acid content is highest in peanut flowers (Yin et al., 2013). We also found that linoleic acid metabolism was significantly enriched during flower organ development, but the specific relationship between linoleic acid and flower organ development requires further study.

We found no significant differences between flower bud differentiation stages 1 and 5 in the expression of most of the Chinese cabbage flowering genes, and their expression

Genetics and Molecular Research 16 (1): gmr16019233 
patterns were mostly consistent with those observed in Chinese cabbage during flower bud differentiation. Moreover, the fold changes in the DEGs that were related to floral organ development were not large, meaning that the relative difference in floral organ developmental gene expression is not large between flower bud differentiation stages 1 and 5 in pak choi.

\section{Conflicts of interest}

The authors declare no conflict of interest.

\section{ACKNOWLEDGMENTS}

Research supported by the Agricultural Science and Technology Research Plan Project of Shanxi Province (\#20150311010-2, \#20140311009-3 and \#20140311011-4), the National Natural Science Foundation of China (\#31401885) and Natural Science Foundation of Shanxi Province, China (2017).

\section{REFERENCES}

Cardon GH, Höhmann S, Nettesheim K, Saedler H, et al. (1997). Functional analysis of the Arabidopsis thaliana SBP-box gene SPL3: a novel gene involved in the floral transition. Plant J. 12: 367-377. http://dx.doi.org/10.1046/j.1365313X.1997.12020367.X

Chen QZ (2010). Cloning, expression and function analysis of BcNS in Brassica campestris L. Doctoral thesis, Zhejiang University, Zhejiang, Hangzhou, China.

Coen ES and Meyerowitz EM (1991). The war of the whorls: genetic interactions controlling flower development. Nature 353: 31-37. http://dx.doi.org/10.1038/353031a0

Han YY (2011). Cloning and functional confirmation of a novel pollen-specific gene BcMF20 encoding CZHZ zinc finger protein in Brassica campestris L. Doctoral thesis, Zhejiang University, Zhejiang, Hangzhou, China.

Huang L, Cao J, Zhang Y and Ye Y (2007). Characterization of a novel gene, BcMF7, that is expressed preferentially in pollen of Brassica campestris L. ssp. chinensis Makino. Sci. China C Life Sci. 50: 497-504. http://dx.doi.org/10.1007/ $\underline{\text { s11427-007-0056-7 }}$

Huang L, Cao JS, Zhang AH and Ye YQ (2008a). Characterization of a putative pollen-specific arabinogalactan protein gene, BcMF8, from Brassica campestris ssp. chinensis. Mol. Biol. Rep. 35: 631-639. http://dx.doi.org/10.1007/ $\underline{\text { s11033-007-9133-Z }}$

Huang L, Cao JS, Zhang AH, Zhang YC, et al. (2008b). Characterisation of BcMF10, a novel gene involved in pollen wall development of Brassica rapa ssp. chinensis. Funct. Plant Biol. 35: 1194-1204. http://dx.doi.org/10.1071/FP08006

Huang L, Ye Y, Zhang Y, Zhang A, et al. (2009). BcMF9, a novel polygalacturonase gene, is required for both Brassica campestris intine and exine formation. Ann. Bot. (Lond.) 104: 1339-1351. http://dx.doi.org/10.1093/aob/mcp244

Huang L, Liu Y, Yu X, Xiang X, et al. (2011). A polygalacturonase inhibitory protein gene (BcMF19) expressed during pollen development in Chinese cabbage-pak-choi. Mol. Biol. Rep. 38: 545-552. http://dx.doi.org/10.1007/s11033$\underline{010-0139-6}$

Jiang JX (2014). Identification and functional characterization of microRNAs involved in pollen development in Brassica campestris ssp. chinensis. Doctoral thesis, Zhejiang University, Zhejiang, Hangzhou, China.

Li Y, Cao J, Huang L, Yu X, et al. (2008). BcMF13, a new reproductive organ-specific gene from Brassica rapa. ssp. chinensis, affects pollen development. Mol. Biol. Rep. 35: 207-214. http://dx.doi.org/10.1007/s11033-007-9072-8

Li YF (2011). The expression and function analysis of BcJMJ30 and BcMF22 related to pollen development and fertilization in Chinese cabbage-pak-choi. Doctoral thesis, Zhejiang University, Zhejiang, Hangzhou, China.

Liu LC, Wang YQ, Zhang T, Li H, et al. (2007). Isolation and characterization of the microspore-related gene BcMF4 in Chinese cabbage-pak-choi and its functional identification in Arabidopsis. J. Hortic. Sci. Biotechnol. 82: 133-139. http://dx.doi.org/10.1080/14620316.2007.11512210

Qiu L (2012). The expression analysis of BcAHA8 and functional identification of BcSKS11 during pollen development and pollination in Brassica campestris L. Doctoral thesis, Zhejiang University, Zhejiang, Hangzhou, China.

Genetics and Molecular Research 16 (1): gmr16019233 
Sablowski RW, Moyano E, Culianez-Macia FA, Schuch W, et al. (1994). A flower-specific Myb protein activates transcription of phenylpropanoid biosynthetic genes. EMBO J. 13: 128-137.

Schiefthaler U, Balasubramanian S, Sieber P, Chevalier D, et al. (1999). Molecular analysis of NOZZLE, a gene involved in pattern formation and early sporogenesis during sex organ development in Arabidopsis thaliana. Proc. Natl. Acad. Sci. USA 96: 11664-11669. http://dx.doi.org/10.1073/pnas.96.20.11664

Scott RJ, Spielman M and Dickinson HG (2004). Stamen structure and function. Plant Cell 16 (Suppl): S46-S60. http:// dx.doi.org/10.1105/tpc. 017012

Winkel-Shirley B (2001). Flavonoid biosynthesis. A colorful model for genetics, biochemistry, cell biology, and biotechnology. Plant Physiol. 126: 485-493. http://dx.doi.org/10.1104/pp.126.2.485

Song JH, Cao JS, Yu XL and Xiang X (2007). BcMF11, a putative pollen-specific non-coding RNA from Brassica campestris ssp. chinensis. J. Plant Physiol. 164: 1097-1100. http://dx.doi.org/10.1016/j.jplph.2006.10.002

Song HX, Fu C, Yin LH, Hou LP, et al. (2015). Cloning and expression analysis of LEAFY homologue in Pak Choi (Brassica rapa subsp. chinensis). Biotechnol. Biotec. Eq. 29: 1035-1042. http://dx.doi.org/10.1080/13102818.201 $\underline{5.1079143}$

Stanko V, Giuliani C, Retzer K, Djamei A, et al. (2014). Timing is everything: highly specific and transient expression of a MAP kinase determines auxin-induced leaf venation patterns in Arabidopsis. Mol. Plant 7: 1637-1652. http://dx.doi. org $/ 10.1093 / \mathrm{mp} / \mathrm{ssu} 080$

Sun M, Qi X, Hou L, Xu X, et al. (2015). Gene expression analysis of pak choi in response to vernalization. PLoS One 10: e0141446. http://dx.doi.org/10.1371/journal.pone.0141446

Tang LL, Song YP and Li JK (2013). Advance on molecular mechanism of floral organ development. Acta Bot. Bor-Occ. Sin 335: 1063-1070.

Thompson EP, Wilkins C, Demidchik V, Davies JM, et al. (2010). An Arabidopsis flavonoid transporter is required for anther dehiscence and pollen development. J. Exp. Bot. 61: 439-451. http://dx.doi.org/10.1093/jxb/erp312

Unte US, Sorensen AM, Pesaresi P, Gandikota M, et al. (2003). SPL8, an SBP-box gene that affects pollen sac development in Arabidopsis. Plant Cell 15: 1009-1019. http://dx.doi.org/10.1105/tpc.010678

Wang YQ (2003). Sterility A/B line and isolation of genes related to microspore development in Chinese Cabbage-pak-choi (Brassica campestris L. ssp. chinensis Makino). Doctoral thesis, Zhejiang University, Zhejiang, Hangzhou, China.

Wang YQ, Ye WZ, Cao JS, Yu XL, et al. (2005). Cloning and characterization of the microspore development-related gene BcMF2 in Chinese cabbage pak-choi (Brassica campestris L. ssp. chinensis Makino). J. Integr. Plant Biol. 47: 863872. http://dx.doi.org/10.1111/j.1744-7909.2005.00101.x

Xu ZH (1999). Plant development and reproduction: advance and prospective. Acta Bot. Sin. 41: 909-920.

Xu ZH and Liu CM (1998). Molecular mechanism of plant development. Science Publishing Company, Beijing, 89-104.

Yamaguchi A, Wu MF, Yang L, Wu G, et al. (2009). The microRNA-regulated SBP-Box transcription factor SPL3 is a direct upstream activator of LEAFY, FRUITFULL, and APETALA1. Dev. Cell 17: 268-278. http://dx.doi.org/10.1016/j. devcel.2009.06.007

Yang WC, Ye D, Xu J and Sundaresan V (1999). The SPOROCYTELESS gene of Arabidopsis is required for initiation of sporogenesis and encodes a novel nuclear protein. Genes Dev. 13: 2108-2117. http://dx.doi.org/10.1101/ gad.13.16.2108

Yin DM, Hu XF, Zhang XG, Song JJ, et al. (2013). Temporal and expression of oleate desaturase gene with different O/L values in peanut varieties. Chin. J. Oil Crop Sci. 35: 137-141.

Yu XL (2002). Functional confirmation of a male sterility gene CYP86MF and creation of artificial male sterile lines in Chinese cabbage (Brassica campestris L. ssp. chinensis Makino). Doctoral thesis, Zhejiang University, Zhejiang, Hangzhou, China.

Yu XL, Cao JS, Ye WZ and Wang YQ (2004). Construction of antisense gene CYP86MF plasmid vector and obtaining male sterile transformant by pollen-tube-mediating method. J. Hortic. Sci. Biotechnol. 79: 833-839. http://dx.doi.or $\mathrm{g} / 10.1080 / 14620316.2004 .11511851$

Zhang Q, Su YQ, Yang FX, Peng JN, et al. (2007a). Antioxidative activity of water extracts from leaf, male flower, raw cortex and fruit of Eucommia ulmoides Oliv. For. Prod. J. 57: 74-78.

Zhang Q, Cao J, Liu H, Huang L, et al. (2008). Characterization and functional analysis of a novel PCP gene BcMF5 from Chinese cabbage (Brassica campestris L. ssp. chinensis Makino). J. Plant Physiol. 165: 445-455. http://dx.doi. org/10.1016/j.jplph.2007.06.020

Zhang Y, Schwarz S, Saedler H and Huijser P (2007b). SPL8, a local regulator in a subset of gibberellin-mediated developmental processes in Arabidopsis. Plant Mol. Biol. 63: 429-439. http://dx.doi.org/10.1007/s11103-006-9099-6

Zhang ZX (2011). The expression and function analysis of pollen-specific gene BcSKS11 during pollen development and pollination process in Brassica campestris L. Doctoral thesis, Zhejiang University, Zhejiang, Hangzhou, China.

Genetics and Molecular Research 16 (1): gmr16019233 